University of Wollongong

Research Online

Australian Institute for Innovative Materials -

Papers

Australian Institute for Innovative Materials

$1-1-2011$

Direct Observation of Nodes and Twofold Symmetry in FeSe

Superconductor

Canli Song

Tsinghua University, Chinese Academy of Sciences

Yi-Lin Wang

Chinese Academy Of Sciences

Peng Cheng

Tsinghua University, pc175@uow.edu.au

Yeping Jiang

Tsinghua University, Chinese Academy of Sciences

Wei Li

Tsinghua University

See next page for additional authors

Follow this and additional works at: https://ro.uow.edu.au/aiimpapers

Part of the Engineering Commons, and the Physical Sciences and Mathematics Commons

Research Online is the open access institutional repository for the University of Wollongong. For further information contact the UOW Library: research-pubs@uow.edu.au 


\title{
Direct Observation of Nodes and Twofold Symmetry in FeSe Superconductor
}

\author{
Abstract \\ We investigated the electron-pairing mechanism in an iron-based superconductor, iron selenide (FeSe), \\ using scanning tunneling microscopy and spectroscopy. Tunneling conductance spectra of stoichiometric \\ FeSe crystalline films in their superconducting state revealed evidence for a gap function with nodal lines. \\ Electron pairing with twofold symmetry was demonstrated by direct imaging of quasiparticle excitations \\ in the vicinity of magnetic vortex cores, Fe adatoms, and Se vacancies. The twofold pairing symmetry was \\ further supported by the observation of striped electronic nanostructures in the slightly Se-doped \\ samples. The anisotropy can be explained in terms of the orbital-dependent reconstruction of electronic \\ structure in FeSe. \\ Keywords \\ observation, nodes, direct, twofold, superconductor, symmetry, fese \\ Disciplines \\ Engineering | Physical Sciences and Mathematics \\ Publication Details \\ Song, C., Wang, Y., Cheng, P., Jiang, Y., Li, W., Zhang, T., Li, Z., He, K., Wang, L., Jia, J., Hung, H., Wu, C., Ma, \\ X., Chen, X. \& Xue, Q. (2011). Direct Observation of Nodes and Twofold Symmetry in FeSe \\ Superconductor. Science, 332 1410-1413.

\section{Authors} \\ Canli Song, Yi-Lin Wang, Peng Cheng, Yeping Jiang, Wei Li, Tong Zhang, Zhi Li, Ke He, Lili Wang, Jin-Feng \\ Jia, Hsiang-Hsuan Hung, Congjun Wu, Xu-Cun Ma, Xi Chen, and Qi-Kun Xue
}


onances in dependence on $H$. When $H$ decreases, resonances I and II show opposite spectral behavior, and the transmittance intensities of the two resonance branches cross one another. In particular, resonance I evolves from a broad profile to a narrower and suppressed resonance, whereas resonance II grows in strength and becomes more and more pronounced. This is due to the fact that when the middle rod is successively shifted toward the bottom shorter rod pair and therefore couples more strongly to it, resonance II is enhanced. Simultaneously, resonance I is reduced because the middle rod is shifted gradually away from the top longer rod pair and therefore couples less strongly to it. It is noteworthy that the spectral positions of the two quadrupolar resonances can also provide information on the spatial structure change. Figure $4 \mathrm{C}$ shows the calculated spectral positions of the two quadrupolar resonances in dependence of $H$. When the middle rod is shifted downward (see the gray region), the position of resonance I stays nearly the same, whereas resonance II shifts to lower energies. In contrast, when the middle rod is shifted upward (see the white region), resonance II does not show a prominent position change, whereas resonance I shifts to significantly lower energies. Figure 4 clearly demonstrates how the full spectral behavior of the $3 \mathrm{D}$ plasmonic structure is correlated to the structural changes in space and will allow for evaluation of the magnitudes as well as the directions of structural changes. Differential spectra that evaluate $3 \mathrm{D}$ conformational changes from the initial configuration to successive configurations offer even more distinct spectral features and facilitate the identification of specific motions. Figures S4 and S5 (17) show how this can aid the spectral analysis of the 3D ruler system upon minute structural tilting and twisting.

To demonstrate the fundamental concepts of 3D plasmon rulers, we used in our study a complex sequence of nanolithography steps. The resulting optical response of the 3D plasmon ruler has been correlated with the particle plasmon resonances of the individual nanostructure assembly. The same concepts can be applied to single metallic nanocrystals joined together by oligonucleotides or peptides (21-24), giving rise to a new generation of plasmon rulers with unprecedented ability to monitor the sequence of events that occur during a wide variety of macromolecular transformations in three dimensions. Metallic nanoparticles of different lengths or sizes could be attached at different positions of the DNA or protein $(25,26)$, and each metallic element may move individually or collectively in three dimensions. Dark-field microspectroscopy of the scattering or the extinction spectrum would offer a useful tool to identify the 3D arrangement of the different constituents in real time, as it is unambiguously correlated to very distinct and rich spectral features. As in the case of nuclear magnetic resonance, 3D plasmon rulers could use a lookup database where the optical spectra cor- responding to all possible structural configurations are stored. Spectral features can then be associated with certain distortions. Novel methods of data mining and inference would facilitate this task. This concept can be further extended by using polarization sensitivity as well as tomography-like spectroscopy from different directions. The realization of 3D plasmon rulers using nanoparticles and biochemical linkers is challenging, but 3D nanoparticle assemblies with desired symmetries and configurations have been successfully demonstrated very recently $(24,25,27-30)$. These exciting experimental achievements will pave the road toward the realization of $3 \mathrm{D}$ plasmon rulers in biological and soft-matter systems.

\section{References and Notes}

1. C. Sönnichsen, B. M. Reinhard, J. Liphardt, A. P. Alivisatos, Nat. Biotechnol. 23, 741 (2005).

2. G. L. Liu et al., Nat. Nanotechnol. 1, 47 (2006).

3. Y. W. Jun et al., Proc. Natl. Acad. Sci. U.S.A. 106, 17735 (2009).

4. L. Stryer, Annu. Rev. Biochem. 47, 819 (1978).

5. J. A. Fan et al., Science 328, 1135 (2010).

6. M. Hentschel et al., Nano Lett. 10, 2721 (2010).

7. J. B. Lassiter et al., Nano Lett. 10, 3184 (2010).

8. C. Sönnichsen, A. P. Alivisatos, Nano Lett. 5, 301 (2005).

9. S. Zhang, D. A. Genov, Y. Wang, M. Liu, X. Zhang, Phys. Rev. Lett. 101, 047401 (2008).

10. N. Liu et al., Nat. Mater. 8, 758 (2009).

11. N. Liu et al., Nano Lett. 10, 1103 (2010).

12. N. Papasimakis, V. A. Fedotov, N. I. Zheludev, S. L. Prosvirnin, Phys. Rev. Lett. 101, 253903 (2008).

13. P. Tassin, L. Zhang, T. Koschny, E. N. Economou, C. M. Soukoulis, Phys. Rev. Lett. 102, 053901 (2009).

14. N. Liu et al., Nat. Mater. 7, 31 (2008).

15. G. Granet, ]. P. Plumey, ]. Opt. A 4, S145 (2002).
16. M. A. Ordal et al., Appl. Opt. 22, 1099 (1983).

17. See supporting material on Science Online.

18. C. L. G. Alzar, M. A. G. Martinez, P. Nussenzveig, Am. J. Phys. 70, 37 (2002).

19. M. Fleischhauer, A. Imamoglu, J. P. Marangos, Rev. Mod. Phys. 77, 633 (2005).

20. H. Xu, Y. Lu, Y. Lee, B. S. Ham, Opt. Express 18, 17736 (2010).

21. C. D. Mao, W. Q. Sun, N. C. Seeman, Nature 386, 137 (1997).

22. E. Winfree, F. R. Liu, L. A. Wenzler, N. C. Seeman, Nature 394, 539 (1998)

23. J. P. Zheng et al., Nature 461, 74 (2009).

24. S. J. Tan, M. J. Campolongo, D. Luo, W. Cheng, Nature Nanotechnol., 10.1038/nnano.2011.49 (2011).

25. A. J. Mastroianni, S. A. Claridge, A. P. Alivisatos, J. Am. Chem. Soc. 131, 8455 (2009).

26. J. Sharma et al., Science 323, 112 (2009).

27. P. Cigler, A. K. R. Lytton-Jean, D. G. Anderson, M. G. Finn, S. Y. Park, Nat. Mater. 9, 918 (2010).

28. M. R. Jones et al., Nat. Mater. 9, 913 (2010).

29. J. W. Zheng et al., Nano Lett. 6, 1502 (2006).

30. D. R. Han et al., Science 332, 342 (2011).

Acknowledgments: We thank T. Pfau and M. Dressel for useful discussions and comments and $\mathrm{S}$. Hein for his material visualizations. N.L. and A.P.A. were supported by the NIH Plasmon Rulers Project, grant NIH NOT-OD-09-056. M.H., T.W., and H.G. were financially supported by the Deutsche Forschungsgemeinschaft (DFG) (grants SPP1391 and FOR557), by The German Ministry of Science (grants 13 N9048 and 13N10146), and by Landesstiftung B. W.T.W. were also supported by the DFG (grant GI 269/11-1) and by DeutschFranzösische Hochschule-Université franco-allemande.

\section{Supporting Online Material}

www.sciencemag.org/cgi/content/full/332/6036/1407/DC1 Materials and Methods

Figs. S1 to S5

3 November 2010; accepted 12 May 2011

$10.1126 /$ science. 1199958

\section{Direct Observation of Nodes and Twofold Symmetry in FeSe Superconductor}

Can-Li Song, ${ }^{1,2}$ Yi-Lin Wang, ${ }^{2}$ Peng Cheng, ${ }^{1}$ Ye-Ping Jiang, ${ }^{1,2}$ Wei Li, ${ }^{1}$ Tong Zhang, ${ }^{1,2}$ Zhi Li, $^{2}$ $\mathrm{Ke} \mathrm{He}^{2}{ }^{2}$ Lili Wang, ${ }^{2}$ jin-Feng Jia, ${ }^{1}$ Hsiang-Hsuan Hung, ${ }^{3}$ Congjun Wu, ${ }^{3}$ Xucun $\mathrm{Ma}^{2}{ }^{2}{ }{ }^{2}$ Xi Chen, ${ }^{1 *}$ Qi-Kun Xue ${ }^{1,2}$

We investigated the electron-pairing mechanism in an iron-based superconductor, iron selenide (FeSe), using scanning tunneling microscopy and spectroscopy. Tunneling conductance spectra of stoichiometric FeSe crystalline films in their superconducting state revealed evidence for a gap function with nodal lines. Electron pairing with twofold symmetry was demonstrated by direct imaging of quasiparticle excitations in the vicinity of magnetic vortex cores, Fe adatoms, and Se vacancies. The twofold pairing symmetry was further supported by the observation of striped electronic nanostructures in the slightly Se-doped samples. The anisotropy can be explained in terms of the orbital-dependent reconstruction of electronic structure in FeSe.

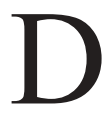
espite intense experimental investigation, the pairing symmetry in the recently discovered iron $(\mathrm{Fe})$-based superconductors remains elusive (1-3). Phonon-mediated pairing in conventional superconductors is typically isotropic, leading to $s$-wave symmetry. Unconventional pairing mechanisms, such as spin fluctuations, may give rise to an order parameter with its sign change over the Fermi surfaces and a pairing symmetry such as $s \pm(4,5)$. The $s \pm$ scenario is supported by the phase-sensitive
"Josephson tunneling" $(6,7)$ and angle-resolved photoemission spectroscopy $(8)$ experiments. If the sign change occurs on a single electron or

${ }^{1}$ State Key Laboratory for Low-Dimensional Quantum Physics, Department of Physics, Tsinghua University, Beijing 100084, the People's Republic of China. 'Institute of Physics, Chinese Academy of Sciences, Beijing 100190, the People's Republic of China. ${ }^{3}$ Department of Physics, University of California, San Diego, La Jolla, CA 92093-0319, USA

*To whom correspondence should be addressed. E-mail: xcma@aphy.iphy.ac.cn (X.M.); xc@mail.tsinghua.edu.cn (X.C.) 
hole pocket, then nodes should show up in the superconducting gap function (5). The presence of nodes in the Fe-based superconductors is still very controversial $(9-12)$. Here, we report the observation of nodal superconductivity in iron selenide (FeSe) by use of a low-temperature scanning tunneling microscope (STM). We find that the symmetry of the order parameter is twofold instead of fourfold.

FeSe is the simplest Fe-based superconductor with an ambient-pressure transition temperature of $T_{\mathrm{c}} \sim 8 \mathrm{~K}$ that can increase to $37 \mathrm{~K}$ at a pressure of $8.9 \mathrm{GPa}(1,2)$. However, the uncertainty in the stoichiometry of $\mathrm{Fe}(\mathrm{Se}, \mathrm{Te})$ samples $(1-3)$ has made it challenging to understand the superconducting and normal states in the materials. To avoid this complexity, we grew the stoichiometric FeSe single-crystalline films on the $\mathrm{SiC}(0001)$ substrate with molecular beam epitaxy (MBE) in ultra-high vacuum (UHV) (13) and performed the STM experiment on the films in the same UHV system. The MBE growth of the FeSe films is characterized by a typical layer-by-layer mode, as demonstrated in fig. S1. The STM topographic images (Fig. 1, A and B, and fig. S1) revealed atomically flat and defect-free Se-terminated (001) surfaces with large terraces. The selenium atom spacing of the $(1 \times 1)-$ Se lattice (Fig. 1B) in the topmost layer was $3.8 \AA$, which is in good agreement with a previous report (1). The synchrotron $\mathrm{x}$-ray power diffraction exhibited a structural transition from tetragonal to orthorhombic symmetry at $90 \mathrm{~K}$ for FeSe (14). In the low-temperature orthorhombic phase, the Fe-Fe lattice's constant difference between the two close-packed directions was $0.012 \AA$ at $20 \mathrm{~K}$. This difference is too small to be resolved with STM, so Fig. 1B appears as a square lattice.

The scanning tunneling spectroscopy (STS) probes the quasiparticle density of states and measures the superconducting gap at the Fermi energy $\left(E_{\mathrm{F}}\right)(15)$. In Fig. 1C, we show the tunneling spectra on the sample in Fig. 1A at various temperatures. The spatial homogeneity of the STS spectra (fig. S2) further demonstrates the high quality of the MBE samples. At a temperature below $T_{\mathrm{c}}$, the spectra exhibit two conductance peaks and a gap centered at the Fermi energy. The maximum of the superconducting gap $\Delta_{0}=2.2 \mathrm{meV}$ is half of the energy between the two conductance peaks. The most striking feature of the spectra at $0.4 \mathrm{~K}$, analogous to the cuprate high- $T_{\mathrm{c}}$ superconductors (15), is the V-shaped $d I / d V$ and the linear dependence of the quasiparticle density of states on energy near $E_{\mathrm{F}}$. This feature explicitly reveals the existence of line nodes in the superconducting gap function. At elevated temperatures, the V-shaped spectra in Fig. 1C smear out as the superconducting gap disappears above $T_{\mathrm{c}}$.

We suggest that the nodal superconductivity exists only in FeSe with a composition close to stoichiometry. By introducing Te into the compound, the ternary $\mathrm{Fe}(\mathrm{Se}, \mathrm{Te})$ becomes a nodeless $s \pm$-wave superconductor, which is characterized by a fully gapped tunneling spectrum in the low- temperature limit (16). The nodes are intrinsic to the superconducting gap function of the stoichiometric FeSe. The scattering-induced extrinsic origin of the $\mathrm{V}$-shaped spectrum in FeSe is quite unlikely. If the scattering strength is too weak, the gap is not closed; if it is too strong, there is a finite residual density of states at the Fermi level. In this extrinsic scenario, the V-shaped spec- trum without residual density of states at the Fermi level is only possible in an accidental case in which scattering strength exactly matches a specific value (17).

Examination of the electronic structure in the Brillouin zone (BZ) reveals the origin of the nodes as well as the symmetry of the order parameter. In the unfolded BZ of FeSe (Fig. 1D),
Fig. 1. STM characterization of the as-grown FeSe films. (A) Topographic image ( $2.5 \mathrm{~V}, 0.1 \mathrm{nA}, 200$ by $200 \mathrm{~nm}^{2}$ ) of a FeSe film ( 30 unit cells thick). The step height is $5.5 \AA$. (Inset) The crystal structure. (B) Atomic-resolution STM topography $(10 \mathrm{mV}, 0.1$ $\mathrm{nA}, 5$ by $5 \mathrm{~nm}^{2}$ ) of FeSe film. The bright spots correspond to the Se atoms in the top layer. $a$ and $b$ correspond to either of Fe-Fe bond directions. The same convention is used for $a$ and $b$ axes throughout. (C) Temperature dependence of differential conductance spectra (setpoint, $10 \mathrm{mV}, 0.1 \mathrm{nA}$ ). (D) Schematic of the unfolded Brillouin zone and the Fermi surface (green ellipses). The nodal lines for $\cos k_{x} \cos k_{y}$ and $\left(\cos k_{x}+\right.$
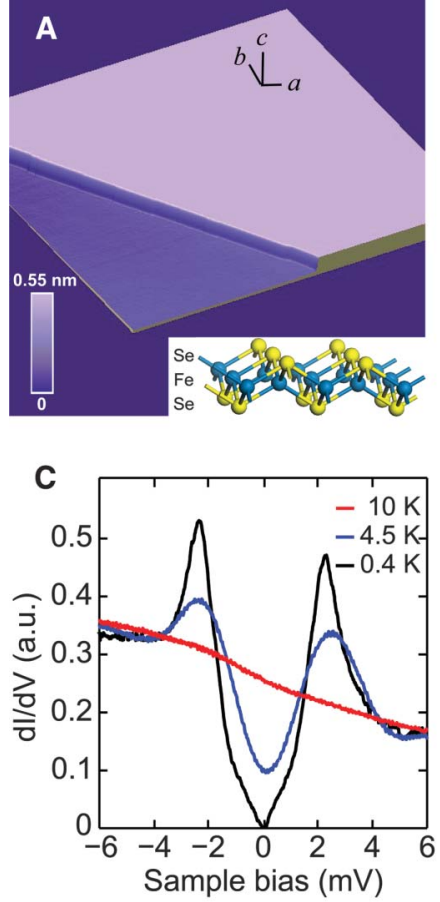

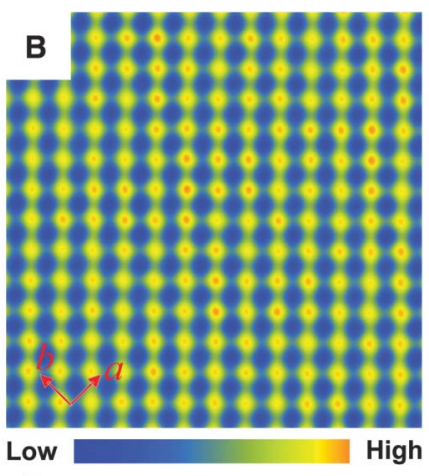

D

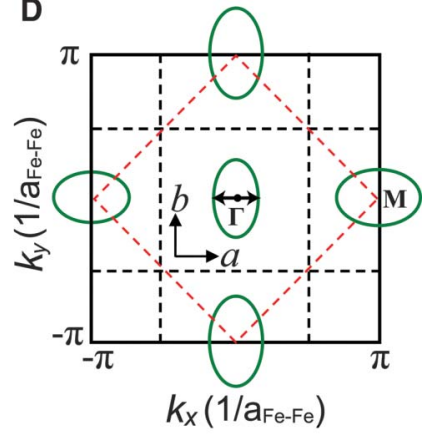

$\cos k_{y}$ ) gap functions are indicated by black and red dashed lines, respectively. The sizes of all pockets are exaggerated for clarity. The black arrow indicates the direction of nesting.
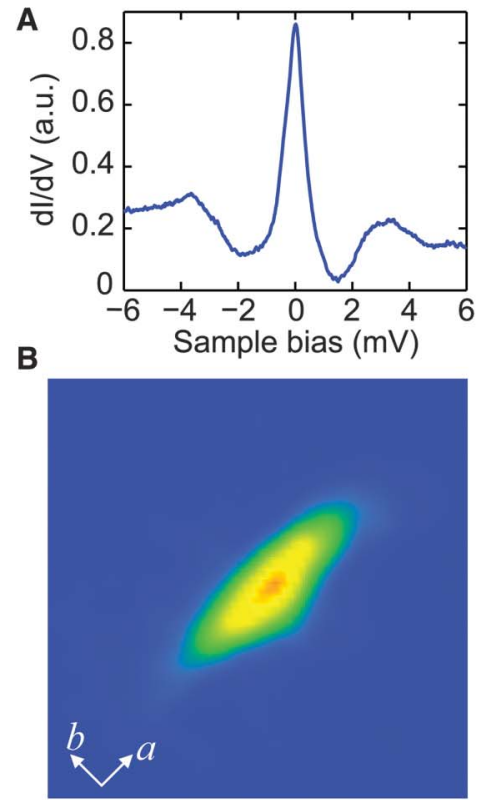

Low

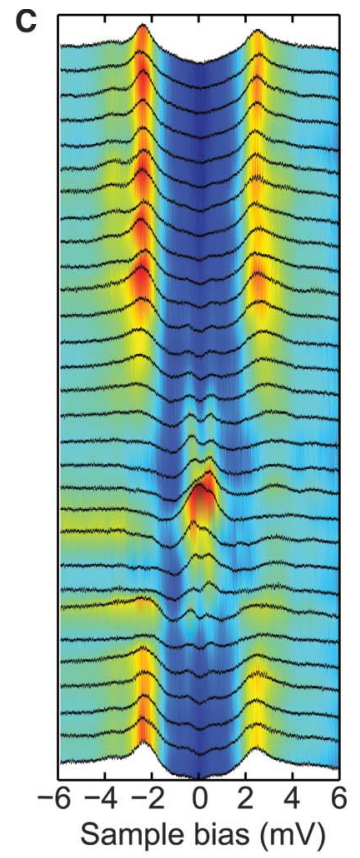

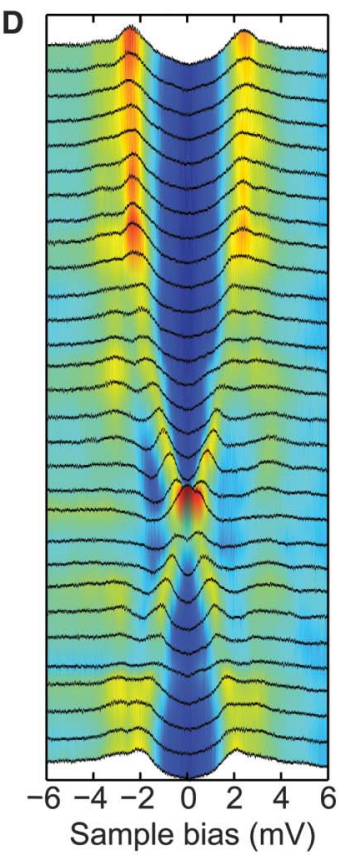

Fig. 2. The vortex core states. (A) STS (setpoint, $10 \mathrm{mV}, 0.1 \mathrm{nA}$ ) on the center of a vortex core. (B) Zero-bias conductance map $\left(40 \times 40 \mathrm{~nm}^{2}\right.$; setpoint, $\left.10 \mathrm{mV}, 0.1 \mathrm{nA}\right)$ for a single vortex at $0.4 \mathrm{~K}$ and $1 \mathrm{~T}$ magnetic field. (C and D) Tunneling conductance curves measured at equally spaced $(2 \mathrm{~nm})$ distances along $a$ and $b$ axes. 
the hole and electron pockets are centered at the $\Gamma[k=(0,0)]$ and $\mathrm{M}[k=(0, \pm \pi)$ and $( \pm \pi, 0)]$ points, respectively (18). The five-band model (19) suggests that the electron pockets at $(0, \pm \pi)$ are mainly derived from the $d_{x z}$ and $d_{x y}$ orbitals of Fe and those at $( \pm \pi, 0)$ from the $d_{y z}$ and $d_{x y}$ orbitals. The hole pockets at $(0,0)$ are derived from the $d_{x z}$ and $d_{y z}$ orbitals. In real space, the nodeless and nodal $s \pm$-wave gap correspond to the pairing between electrons on the next-nearestneighbor $(\mathrm{NNN})$ and nearest-neighbor $(\mathrm{NN}) \mathrm{Fe}$ atoms, respectively. For the nodeless $s \pm$ pairing, the Se-mediated exchange interaction $J_{2}$ between the NNN Fe sites dominates, giving rise to the formation of spin-density wave (SDW) and the full sign reversal between the superconducting gap functions on the hole and electron pockets. However, if the exchange interaction $J_{1}$ between the $\mathrm{NN} \mathrm{Fe}$ sites is comparable with $J_{2}$ then nodes may develop on the electron pockets (the nodal $s \pm$-wave) to minimize the total energy of the system (5).
In the extended $s \pm$-wave model, the gap function is given by

$$
\Delta_{s \pm}=\Delta_{1} \cos k_{x} \cos k_{y}+\Delta_{2}\left(\cos k_{x}+\cos k_{y}\right)
$$

The nodal lines of both $\cos k_{x} \cos k_{y}$ and $\left(\cos k_{x}+\right.$ $\cos k_{y}$ ) are shown in Fig. 1D. According to the local density approximation calculation (18), the radius of the pockets at $M$ points is not large enough to intercept with the nodal lines of $\cos k_{x} \cos k_{y}$, whereas $\left(\cos k_{x}+\cos k_{y}\right)$ can naturally lead to the nodes of superconducting gap on the electron pockets in the two orthogonal $\mathrm{Fe}-\mathrm{Fe}$ directions. The gap function of FeSe is expected to contain the main features of $\left(\cos k_{x}+\cos k_{y}\right)$, implying a stronger exchange interaction between $\mathrm{NN}$ than NNN. This scenario is also consistent with the absence of $(\pi, 0)$ nesting-driven SDW order in FeSe (2). Both $\cos k_{x} \cos k_{y}$ and $\left(\cos k_{x}+\cos k_{y}\right)$ belong to the same representation of the lattice point group; thus, they naturally coexist. Although the $d$-wavelike pairing cannot be ruled out on the basis of our
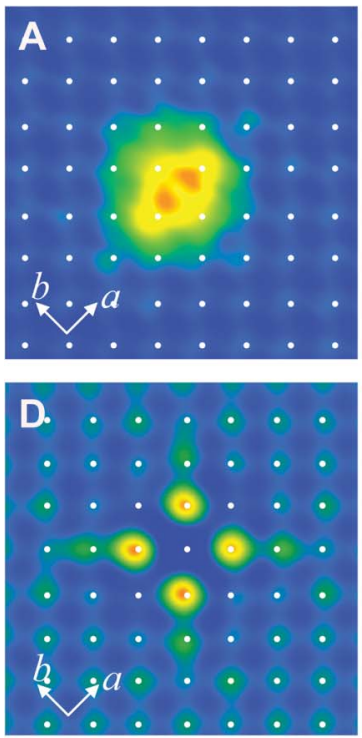

Low
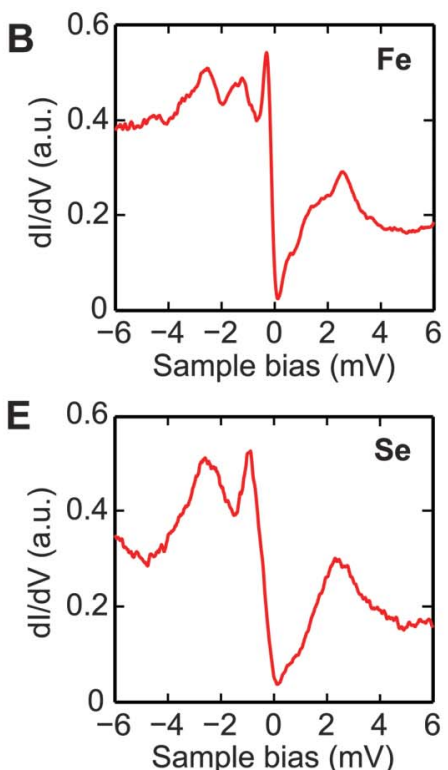
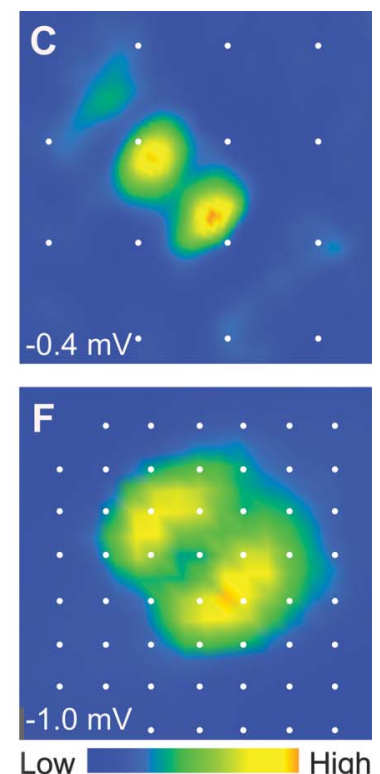

Fig. 3. Impurity-induced bound states in superconducting gap. (A to C) STM topography (10 mV, $0.1 \mathrm{nA}$, 3 by $\left.3 \mathrm{~nm}^{2}\right)$, dl/dV spectrum $(0.4 \mathrm{~K}$; setpoint, $10 \mathrm{mV}, 0.1 \mathrm{nA})$, and density of states map $(-0.4 \mathrm{mV}, 0.1 \mathrm{nA}$, 1.5 by $1.5 \mathrm{~nm}^{2}$ ) of a single Fe adatom. (D to F) STM topography $\left(10 \mathrm{mV}, 0.1 \mathrm{nA}, 3\right.$ by $\left.3 \mathrm{~nm}^{2}\right)$, dl/dV spectrum $(0.4 \mathrm{~K}$; setpoint, $10 \mathrm{mV}, 0.1 \mathrm{nA})$, and density of states map $\left(-1.0 \mathrm{mV}, 0.1 \mathrm{nA}, 3\right.$ by $\left.3 \mathrm{~nm}^{2}\right)$ of a single Se vacancy. The white dots indicate the topmost Se atoms. For each impurity type, at least five impurities were measured, and the energies of the bound states were found to be reproducible within an error of $0.1 \mathrm{meV}$.

Fig. 4. Unidirectional electronic nanostructure induced by extra Se doping. (A) STM topography (10 mV, $0.1 \mathrm{nA}, 40$ by $40 \mathrm{~nm}^{2}$ ) of a Se-rich sample. The unidirectional stripes are along the $b$ axis. The bright features, which are elongated along the diagonal directions of $a$ and $b$ axes, are due to the extra Se atoms. (B)
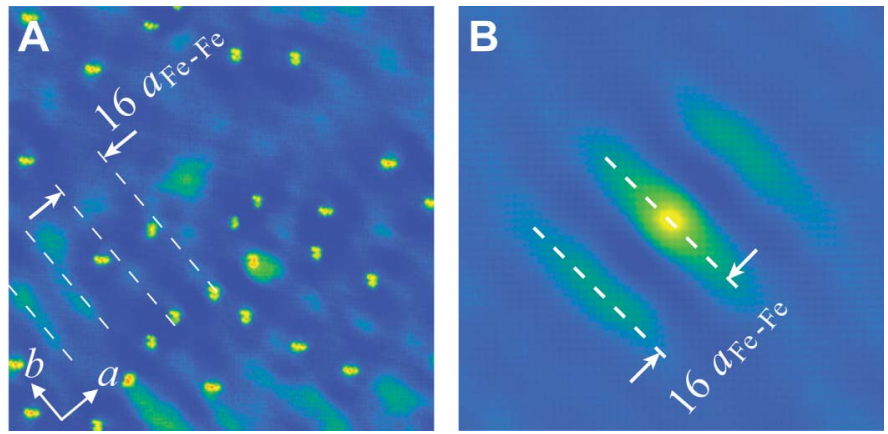

Autocorrelation analysis of the STM image in (A).

current experimental results, the extended $s \pm$-wave pairing is much more natural, considering other evidence for the materials in the same family (16).

The reason why the Cooper pairing is nodal in $\mathrm{FeSe}$ but nodeless in $\mathrm{Fe}(\mathrm{Se}, \mathrm{Te})$ remains a theoretical challenge. A possible mechanism involves the different chalcogen-height $h_{\mathrm{ch}}$ measured from the $\mathrm{Fe}$ plane in $\mathrm{FeSe}$ and $\mathrm{Fe}(\mathrm{Se}, \mathrm{Te})$. Previous studies have shown that in pnictide superconductors, the pnictogen height $h_{\mathrm{pn}}$ can act as a switch from the high- $T_{\mathrm{c}}$ nodeless pairing with large $h_{\mathrm{pn}}$ (for example, $\mathrm{LaFeAsO}$ ) to the low- $T_{\mathrm{c}}$ nodal pairing with small $h_{\mathrm{pn}}$ (for example, LaFePO) (20). FeSe has the smallest $h_{\mathrm{ch}}(1.55 \AA)$ among the $\mathrm{FeSe}_{x} \mathrm{Te}_{1-x}$ compounds. We expect that the small chalcogen-height in FeSe enhances the exchange interaction between the nearest-neighbor Fe atoms and results in a dominant $\left(\cos k_{x}+\cos k_{y}\right)$ pairing symmetry with nodes on the electron pockets.

We generated inhomogeneity in the superconducting state using magnetic field and impurities to gain further insight into the pairing symmetry. When a magnetic field was applied (perpendicularly) to the FeSe sample surface, the field could enter the superconductor in the form of vortices (fig. S3). The superconducting order parameter is zero at the center of a vortex and approaches the zero field value at a distance in the order of coherence length $\xi$. Low-energybound states exist in the vortex because of the constructive interference of repeated Andreev scatterings at the boundary between the normal and superconducting states $(21,22)$. In the case of a superconductor with nodes, the quasiparticles form resonance states instead.

The $d I / d V$ curve at center of a vortex in $\mathrm{FeSe}$ showed a pronounced zero-bias peak (Fig. 2A), in contrast to that in $\mathrm{BaFe}_{1.8} \mathrm{Co}_{0.2} \mathrm{As}_{2}$ (23). The appearance of such a peak demonstrates the high quality of the MBE-grown FeSe films (13). The spatial distribution of the peak reflected the quasiparticle wave function and was mapped out by measuring $d I / d V$ at zero bias in the vicinity of a single vortex (Fig. 2B). This resonance state elongates along the $a$ axis (presumably a direction with nodes). Intuitively, anisotropic distribution of the core state can be understood by the difference between coherence lengths $\xi$ along the $a$ and $b$ directions, which mainly stems from the twofold symmetry of the gap function. Away from the center of a vortex core, the resonance peak splits into two symmetric branches in energy (Fig. 2, C and D). Although the peaks along the $b$ axis eventually merge into the gap edges at a distance of $20 \mathrm{~nm}$ from the center (Fig. 2D), the energy of the peaks along the $a$ axis approaches to $\pm 0.6 \mathrm{meV}$ instead of $\Delta_{0}=2.2 \mathrm{meV}$ (Fig. 2C).

The above features are rather similar to the conventional $s$-wave superconductor $\mathrm{NbSe}_{2}(22)$, in which the observed sixfold star-shaped local density of states of a single vortex and the directiondependent spectra are attributed to the anisotropic $s$-wave pairing with hexagonal symmetry (24). Further theoretical analysis is needed to fully 
understand the direction-dependent behavior of the resonance peaks in a nodal superconductor.

Not all of the vortex cores orientate along the same direction. Twin boundaries occurred in the crystalline films, and the magnetic vortices were easily pinned at these boundaries. As shown in fig. S4, two sides of a twin boundary exhibited different orientations of the vortex cores. Across the boundary (fig. S4, red arrows), the elongation direction of the core states was rotated by $90^{\circ}$. If a sample is composed of twinned domains, the twofold pairing symmetry cannot be revealed by macroscopic probes.

The response of a superconductor to impurities provides another method for uncovering the nature of superconducting pairing symmetry (15). The twofold symmetry of the FeSe gap function is further supported by the impurity-induced resonance states inside the superconducting gap. We deposited Fe atoms (Fig. 3A) on FeSe surface at low temperature $(50 \mathrm{~K})$. Single Fe adatoms formed and occupied hollow sites of the surface Se lattice. On a Fe adatom, two resonance states (at $-1.4 \mathrm{meV}$ and $-0.4 \mathrm{meV}$ ) were clearly observed in STS in Fig. 3B. The density of states map in Fig. 3C again shows the twofold symmetry, but the state is more visible in the direction perpendicular to the long axis of a vortex core. Similar spectra and density of states maps were also observed on Se vacancies (Fig. 3, D to F) (13).

The symmetry breaking from $\mathrm{C}_{4}$ to $\mathrm{C}_{2}$ is further supported by the quantum interference pattern of quasiparticles on samples with extra Se atoms deposited on the surface (Fig. 4A and fig. S5B). A typical topographic image at a sample bias of $10 \mathrm{mV}$ is shown in Fig. 4A. The unidirectional stripes with the wave vectors exclusively along the $a$ axis were observed. The measured period is $4.4 \mathrm{~nm}$, which is approximately 16 times the Fe-Fe atom spacing according to autocorrelation analysis (Fig. 4B). Similarly striped nanostructure was recently demonstrated in the lightly doped $\mathrm{Ca}\left(\mathrm{Fe}_{1-\mathrm{x}} \mathrm{Co}_{\mathrm{x}}\right)_{2} \mathrm{As}_{2}$ (25). In FeSe, we suggest that the $\mathrm{C}_{2}$ symmetric hole pockets (implying nesting, as shown in Fig. 1D) is responsible for the observed unidirectional stripes (26) and the twofold symmetry in electron pairing (fig. S6).

The twofold symmetry could arise from the structural transition from tetragonal to orthorhombic phase at $90 \mathrm{~K}(14)$. However, the orthorhombic lattice distortion of $0.012 \AA$ (half a percent of the lattice constant) can only lead to a very small anisotropy $(\sim 3 \%)$ in electronic structure according to the tight binding model, which is not large enough to account for the anisotropy of the vortex core. The relation between the symmetry breaking and antiferromagnetism is not clear either because long-range-ordered antiferromagnetism is not observed in FeSe under ambient pressure (2). As one of the many possible mechanisms, the recently proposed orbital-dependent reconstruction (26-29) lifts the degeneracy between $d_{x z}$ and $d_{y z}$ orbitals of $\mathrm{Fe}$ and may explain the symmetry breaking from $\mathrm{C}_{4}$ to $\mathrm{C}_{2}$ as well as the nested Fermi surface geometry. Nevertheless, our experimental data are still not sufficient enough to support such a scenario, and the origin of $\mathrm{C}_{2}$ symmetry remains an open question.

\section{References and Notes}

1. F. C. Hsu et al., Proc. Natl. Acad. Sci. U.S.A. 105, 14262 (2008).

2. S. Medvedev et al., Nat. Mater. 8, 630 (2009).

3. D. C. Johnston, Adv. Phys. 59, 803 (2010).

4. I. I. Mazin et al., Phys. Rev. Lett. 101, 057003 (2008).

5. K. Kuroki et al., Phys. Rev. Lett. 101, 087004 (2008).

6. X. H. Zhang et al., Phys. Rev. Lett. 102, 147002 (2009).
7. C. T. Chen, C. C. Tsuei, M. B. Ketchen, Z. A. Ren, Z. X. Zhao, Nat. Phys. 6, 260 (2010).

8. H. Ding et al., Europhys. Lett. 83, 47001 (2008)

9. J. D. Fletcher et al., Phys. Rev. Lett. 102, 147001 (2009).

10. J.-Ph. Reid et al., Phys. Rev. B 82, 064501 (2010).

11. J. K. Dong et al., Phys. Rev. Lett. 104, 087005 (2010).

12. C. Michioka et al., Phys. Rev. B 82, 064506 (2010).

13. Materials and methods are available as supporting material on Science Online.

14. T. M. McQueen et al., Phys. Rev. Lett. 103, 057002 (2009).

15. Ø. Fischer, M. Kugler, I. Maggio-Aprile, C. Brthod, C. Renner, Rev. Mod. Phys. 79, 353 (2007) and references therein.

16. T. Hanaguri, S. Niitaka, K. Kuroki, H. Takagi, Science 328, 474 (2010).

17. Y. Bang, H.-Y. Choi, H. Won, Phys. Rev. B 79, 054529 (2009).

18. A. Subedi, L. J. Zhang, D. J. Singh, M. H. Du, Phys. Rev. B 78, 134514 (2008).

19. S. Graser, T. A. Maier, P. J. Hirschfeld, D. J. Scalapino, N. J. Phys. 11, 025016 (2009).

20. K. Kuroki et al., Phys. Rev. B 79, 224511 (2009)

21. J. D. Shore et al., Phys. Rev. Lett. 62, 3089 (1989).

22. H. F. Hess et al., Phys. Rev. Lett. 64, 2711 (1990).

23. Y. Yin et al., Phys. Rev. Lett. 102, 097002 (2009).

24. N. Hayashi et al., Phys. Rev. Lett. 77, 4074 (1996).

25. T. M. Chuang et al., Science 327, 181 (2010).

26. T. Shimojima et al., Phys. Rev. Lett. 104, 057002 (2010)

27. W. C. Lv, J. S. Wu, P. Phillips, Phys. Rev. B 80, 224506 (2009).

28. C.-C. Lee et al., Phys. Rev. Lett. 103, 267001 (2009).

29. C.-C. Chen et al., Phys. Rev. B 82, 100504 (2010).

Acknowledgments: We thank ]. P. Hu, T. Xiang, and X. Dai for helpful discussions. The work was financially supported by National Science Foundation and Ministry of Science and Technology of China. C.J.W. and H.-H.H. are supported by U.S. Army Research Office under award W911NF0810291.

\section{Supporting Online Material}

www.sciencemag.org/cgi/content/full/332/6036/1410/DC1

Materials and Methods

Figs. S1 to S6

References

27 December 2010; accepted 14 April 2011

$10.1126 /$ science. 1202226

\section{Thermal Structure and Dynamics of Saturn's Northern Springtime Disturbance}

Leigh N. Fletcher, ${ }^{1 *}$ Brigette E. Hesman, ${ }^{2}$ Patrick G. J. Irwin, ${ }^{1}$ Kevin H. Baines, ${ }^{3}$ Thomas W. Momary, ${ }^{4}$ Agustin Sanchez-Lavega, ${ }^{5}$ F. Michael Flasar, ${ }^{6}$ Peter L. Read, ${ }^{1}$ Glenn S. Orton, ${ }^{4}$ Amy Simon-Miller, ${ }^{6}$ Ricardo Hueso, ${ }^{5}$ Gordon L. Bjoraker, ${ }^{6}$ Andrei Mamoutkine, ${ }^{6}$ Teresa del Rio-Gaztelurrutia, ${ }^{5}$ ]ose M. Gomez, ${ }^{7}$ Bonnie Buratti, ${ }^{4}$ Roger N. Clark, ${ }^{8}$ Philip D. Nicholson, ${ }^{9}$ Christophe Sotin ${ }^{4}$

Saturn's slow seasonal evolution was disrupted in 2010-2011 by the eruption of a bright storm in its northern spring hemisphere. Thermal infrared spectroscopy showed that within a month, the resulting planetary-scale disturbance had generated intense perturbations of atmospheric temperatures, winds, and composition between $20^{\circ}$ and $50^{\circ} \mathrm{N}$ over an entire hemisphere $(140,000$ kilometers). The tropospheric storm cell produced effects that penetrated hundreds of kilometers into Saturn's stratosphere (to the 1-millibar region). Stratospheric subsidence at the edges of the disturbance produced "beacons" of infrared emission and longitudinal temperature contrasts of 16 kelvin. The disturbance substantially altered atmospheric circulation, transporting material vertically over great distances, modifying stratospheric zonal jets, exciting wave activity and turbulence, and generating a new cold anticyclonic oval in the center of the disturbance at $41^{\circ} \mathrm{N}$.

I n contrast to Jupiter's frequent large-scale storms and vortices (1), Saturn typically apoccurring infrequently at mid-latitudes (2-5). Saturn's temperatures, winds, and circulation patterns above the visible cloud decks evolve slowly in response to the changing levels of sunlight (6), and eruptions of planetary-scale disturbances are extremely rare $(7,8)$, even though convective processes and eddy mixing are thought to play a key role in transporting Saturn's internal heat and maintaining the zonal jets (2). This slow evolution was disrupted on 5 December 2010 by a convective plume of bright cloud material near $40^{\circ} \mathrm{N}$ (planetographic latitude) in Saturn's northern springtime hemisphere, which created

${ }^{1}$ Atmospheric, Oceanic and Planetary Physics, Department of Physics, Clarendon Laboratory, University of Oxford, Parks Road, Oxford, OX1 3PU, UK. 'Department of Astronomy, University of Maryland, College Park, MD 20742, USA. ${ }^{3}$ Space Science and Engineering Center, University of WisconsinMadison, 1225 West Dayton Street, Madison, WI 53706, USA. ${ }^{4}$ Jet Propulsion Laboratory, 4800 Oak Grove Drive, Pasadena, CA 91109, USA. ${ }^{5}$ Departamento de Física Aplicada I, Escuela Técnica Superior Ingenieros, Universidad del País Vasco, Alameda Urquijo s/n, 48013 Bilbao, Spain. ${ }^{6}$ NASA Goddard Space Flight Center, Greenbelt, MD 20771, USA. 'Fundacion Esteve Duran, Barcelona, Spain. ${ }^{8}$ U.S. Geological Survey, MS 964, Box 25046 Federal Center, Denver, CO 80225, USA. ${ }^{\circ}$ Departmentof Astronomy, Cornell University, Space Science Building, Ithaca, NY 14853, USA.

*To whom correspondence should be addressed. E-mail: fletcher@atm.ox.ac.uk 


\section{Science}

\section{Direct Observation of Nodes and Twofold Symmetry in FeSe Superconductor}

Can-Li Song, Yi-Lin Wang, Peng Cheng, Ye-Ping Jiang, Wei Li, Tong Zhang, Zhi Li, Ke He, Lili Wang, Jin-Feng Jia, Hsiang-Hsuan Hung, Congjun Wu, Xucun Ma, Xi Chen and Qi-Kun Xue

Science 332 (6036), 1410-1413.

DOI: $10.1126 /$ science. 1202226

ARTICLE TOOLS

SUPPLEMENTARY

MATERIALS

REFERENCES

PERMISSIONS http://science.sciencemag.org/content/332/6036/1410

http://science.sciencemag.org/content/suppl/2011/06/15/332.6036.1410.DC1

This article cites 28 articles, 3 of which you can access for free http://science.sciencemag.org/content/332/6036/1410\#BIBL

http://www.sciencemag.org/help/reprints-and-permissions

Use of this article is subject to the Terms of Service

Science (print ISSN 0036-8075; online ISSN 1095-9203) is published by the American Association for the Advancement of Science, 1200 New York Avenue NW, Washington, DC 20005. 2017 () The Authors, some rights reserved; exclusive licensee American Association for the Advancement of Science. No claim to original U.S. Government Works. The title Science is a registered trademark of AAAS. 